\title{
CORRELAÇÃO DOS MÉTODOS DE AVALIACCÃO NUTTICIONAL DE PACIENTES SUBMETIDOS À HEMODIÁLISE
}

Marcela Rossini Montenegro

Nutricionista graduada pela Universidade Presbiteriana Mackenzie, Brasil.

\section{Renata Monteiro Walter}

Nutricionista graduada pela Universidade Presbiteriana Mackenzie, Brasil.

\section{Juliana Masami Morimoto}

Doutora em Nutrição em Saúde Pública, Centro de Ciências Biológicas e da Saúde, Universidade Presbiteriana Mackenzie, Brasil.

\section{Ana Carolina Almada Colucci Paternez}

Doutora em Saúde Pública, Centro de Ciências Biológicas e da Saúde, Universidade Presbiteriana Mackenzie, Brasil.

E-mail: ana.colucci@mackenzie.br
RESUMO: A Doença Renal Crônica (DRC) constitui-se da perda lenta, progressiva e irreversível das funções renais excretoras, endócrinas e metabólicas. O objetivo do trabalho foi correlacionar os métodos de avaliação nutricional em pacientes submetidos à hemodiálise em uma clínica particular do município de São Paulo. O estudo foi realizado em uma clínica especializada em doença renal crônica situada no município de São Paulo com pacientes de ambos os sexos e com idade superior a 18 anos, que realizavam hemodiálise no período na coleta de dados. Para a coleta de dados foi aplicada a Avaliação Subjetiva Global para pacientes com doença renal. Foi calculado o índice de Massa Corporal (IMC) e aferidas a dobra cutânea de tríceps (DCT) e circunferência de braço (CB) após a sessão de diálise. Com as medidas aferidas, foi calculada a circunferência muscular do braço (CMB). Calculou-se a correlação de Spearman entre os resultados da avaliação subjetiva e as variáveis antropométricas. A amostra final foi composta por 60 pacientes, $36,6 \%$ do sexo masculino e $63,3 \%$ do sexo feminino, sendo 37 idosos e 23 adultos. A intensidade das correlações entre as variáveis IMC vs. Questionário, IMC vs. $\mathrm{CMB}, \mathrm{DCT}$ vs. $\mathrm{CB}$ e $\mathrm{CMB}$ vs. Questionário se mostrou muito fraca, fraca, fraca e muito fraca respectivamente. Destaca-se que as correlações IMC vs. CMB e DCT vs. $\mathrm{CB}$, apesar de fracas, apresentaram significância estatística $(\mathrm{p}<0,05)$. Conclui-se que o uso de diferentes métodos de avaliação nutricional representa significativa melhoria do padrão diagnóstico de pacientes com DRC.

PALAVRAS-CHAVE: Avaliação Nutricional; Diálise Renal; Insuficiência Renal Crônica.

\section{CO-RELATIONSHIP BET WEEN METHODS IN NUTRITIONAL EVALUATION OF PATIENTS UNDERGOING HEMODIALYSIS}

\begin{abstract}
Chronic Renal Disease (CRD) comprises the slow, progressive and irreversible loss of excretory, endocrine and metabolic renal function. Current analysis co-related the nutritional evaluation methods in patients undergoing hemodialysis in a private clinic in São Paulo, Brazil. The study was performed in a clinic specialized in CRD for over-18-year-old patients of both genders that were undergoing hemodialysis during data collection. Global Subjective Evaluation for patients with kidney diseases was applied. Body Mass Index (BMI), the triceps skin folds (TSF) and arm circumference (AC) were calculated after each session. The arm's muscle circumference (AMC) was calculated and Spearman's co-relationship was established between the results of subjective evaluation and anthropometric variables. Final sample comprised 50 patients, of which $36.6 \%$ were males and $63.3 \%$ were females, with 37 elderly people and 23 adults. Co-relation
\end{abstract}


intensities between BMI and Questionnaire, between $\mathrm{BMI}$ and $\mathrm{AMC}$, TSF and $\mathrm{AC}$, and $\mathrm{AMC}$ and Questionnaire were respectively very weak, weak, weak and very weak. It should be underscored that, albeit weak, BMI v. AMC and TSF v. AC were statistically significant $(p<0.05)$. Results show that the use of different nutritional evaluation methods reveals significant improvement in diagnostic standard in patients with CRD.

KEY WORDS: Nutritional Evaluation; Renal Dialysis; Chronic Renal Non-sufficiency.

\section{INTRODUÇÃO}

A doença renal crônica (DRC) está se tornando uma epidemia e um dos principais problemas de saúde pública em todo o mundo. Segundo dados estimados do Ministério da Saúde, existem no Brasil mais de 30 milhões de hipertensos (24,4\%), 7 milhões de diabéticos (5,8\%), 17 milhões de obesos (13,9\%), 56 milhões de pessoas com sobrepeso $(46,6 \%)$ e 20 milhões com dislipidemia, de um total de população adulta estimada de 120 milhões de pessoas. O aumento do número de indivíduos acometidos pela hipertensão arterial sistêmica (HAS) e pelo diabetes mellitus (DM) está diretamente relacionado com o aumento da incidência e prevalência de DRC (KIRSZTAJN; BASTOS; ANDRIOLO, 2011; BIAVO et al., 2012).

Não existem dados suficientes que ilustrem a magnitude da incidência e prevalência de DRC no Brasil, porém se a realidade norte-americana fosse usada como referência (cerca de 10\% da população apresenta DRC), a estimativa seria de cerca de 20 milhões de brasileiros acometidos (SESSO et al., 2014).

A DRC constitui-se da perda lenta, progressiva e irreversível das funções renais excretoras, endócrinas e metabólicas. Por certo tempo o paciente pode ser assintomático por causa dos processos adaptativos do organismo (OLIVEIRA et al., 2012), que podem ser: reserva funcional, isto é, multiplicação do trabalho dos néfrons remanescentes, alteração na dinâmica renal e adaptação tubular. Tais processos podem levar a uma progressão da lesão renal e como consequência a evolução para fases mais avançadas e sintomáticas da doença (NATIONAL KIDNEY FOUNDATION, 2002).
É diagnosticado com DRC qualquer adulto com idade maior ou igual a 18 anos que, por um período maior ou igual a 3 meses, apresenta filtração glomerular menor que $60 \mathrm{ml} / \mathrm{min} / 1,73 \mathrm{~m}^{2}$, assim como aqueles com filtração glomerular maior que $60 \mathrm{ml} / \mathrm{min} / 1,73 \mathrm{~m}^{2} \mathrm{com}$ alguma evidência de lesão da estrutura renal (SESSO et al., 2014).

Para classificar a DRC, que pode ser dividida em cinco estágios funcionais, necessita-se da presença de anormalidades no sedimento urinário, alteração em exame de imagem renal e determinação da filtração glomerular. O estágio 1 (Lesão Renal com Função Renal Normal) corresponde às fases iniciais da lesão renal, nas quais ocorre a presença de microalbuminúria ou proteinúria e/ou hematúria. No estágio 2 (Lesão Renal com Insuficiência Renal Leve), onde ocorre o início da insuficiência renal, o indivíduo apresenta lesão do parênquima renal. Já no estágio 3 (Lesão Renal com Insuficiência Renal Moderada) os sintomas renais podem estar presentes de forma branda e o paciente geralmente queixa-se dos sintomas das doenças de base como DM e HAS. No estágio 4 (Lesão Renal com Insuficiência Renal Grave) o paciente apresenta sinais e sintomas de uremia como náuseas, vômitos, perda do apetite, etc. E no estágio 5 (Lesão Renal com Insuficiência Renal Terminal) o rim já não é mais capaz de manter as funções mínimas para a sobrevivência do indivíduo (NATIONAL KIDNEY FOUNDATION, 2002).

Em pacientes com DRC devem ser alcançadas metas específicas para pressão arterial, níveis de hemoglobina glicada, colesterol total e frações. O controle de peso, redução do consumo de sal, exercício físico, abandono de tabagismo e controle de estresse psicoemocional podem ser meios não medicamentosos para uma primeira abordagem (KIRSZTAJN; BASTOS; ANDRIOLO, 2011).

Caso os mecanismos adaptativos não consigam remover adequadamente os produtos de degradação metabólica, o tratamento dialítico deve ser iniciado (OLIVEIRA et al., 2012). Este tratamento deve ser realizado por meio de terapia renal substitutiva, através de diálise peritoneal, hemodiálise e transplante renal (FAVA et al., 2006). A diálise peritoneal é indicada para crianças, pacientes que não toleram a hemodiálise e aqueles com impossibilidade de acesso vascular. Há 
indicação preferencial para hemodiálise de pacientes com compleição física grande sem função renal residual, com redução importante da função peritoneal ou extensas aderências abdominais que limitem o fluxo do dialisato, ostomias, hipertrigliceridemia grave, alto risco cardiovascular, falta de estrutura doméstica, etc. Porém, pacientes que apresentam familiares dispostos à doação renal podem ser preparados precocemente para realizar o transplante renal e evitar o tratamento dialítico (KAMIMURA; BAZANELLI; CUPPARI, 2013).

Os pacientes que são submetidos à terapia de substituição renal merecem atenção por apresentarem modificações parácrinas e endócrinas. Essas alterações estão relacionadas aos distúrbios no metabolismo de energia e de macronutrientes, como o aumento do catabolismo proteico, a resistência à insulina e alterações no metabolismo de lipoproteínas. O produto dessas condições certamente contribui para a redução da massa muscular, que facilita a desnutrição energético-proteica (DEP) (NERBASS; CUPPARI, 2013; OLIVEIRA et al., 2010). Diversos estudos mostram que a DEP está presente em cerca de 45 a $55 \%$ dos pacientes em tratamento conservador, 18 a $50 \%$ dos pacientes em diálise peritoneal e 23 a 76\% dos pacientes em hemodiálise (KAMIMURA; BAZANELLI; CUPPARI, 2012). Em razão dessa alta prevalência, fica clara a importância de acompanhamento nutricional e realização de avaliação nutricional para identificar as causas de risco e/ou deterioração do estado nutricional (VEGINE et al., 2011).

A complexa avaliação de um paciente dialítico vem sendo estudada desde a década de 70 , quando surgiram os primeiros estudos identificando a desnutrição como uma condição prevalente nos pacientes com DRC. Entretanto, foi somente na década de 80 que a desnutrição foi identificada como um fator de risco para morbidade e mortalidade nesta população. Desde essa época, vários métodos passaram a ser testados para o diagnóstico nutricional e como antecedentes de desfechos clínicos. Na década de 90 passaram a ser utilizados novos métodos de avaliação nutricional como, por exemplo, a bioimpedância elétrica (CUPPARI; KAMIMURA, 2009).

Nos dias atuais não existe um único marcador que forneça o diagnóstico nutricional de maneira inequívoca e completa, sendo necessário empregar diferentes parâmetros como métodos clínicos, bioquímicos e antropométricos que, analisados em conjunto, permitam a identificação de riscos ou distúrbios nutricionais já instalados (BIGOGNO; FETTER; AVESANI, 2014). Entre os métodos antropométricos, os mais utilizados são o Índice de Massa Corporal (IMC), Circunferência Abdominal, Circunferência de Braço, Circunferência Muscular de Braço, Área Muscular de Braço Corrigida, Área de Gordura do Braço e Prega Cutânea de Tríceps. Já entre os marcadores bioquímicos (reserva de proteínas viscerais), a albumina, pré-albumina, transferrina e creatinina são os mais utilizados (KAMIMURA et al., 2004).

Apesar de o IMC ser um dos métodos antropométricos reconhecidos como adequado marcador de gordura corporal, em pacientes renais os valores podem estar distorcidos em situações de retenção hídrica. Indivíduos com os mesmos valores de IMC podem estar expostos a riscos de formas diferentes, pois além de "mascarar" os resultados em situações de edema, este indicador também não é sensível para detectar depleção proteica e aumento de gordura visceral (CUPPARI; KAMIMURA, 2009). Para avaliação antropométrica de pacientes específicos como os renais, a aferição das medidas deve ser feita logo após a sessão de diálise (5 a 20 minutos), pois este grupo apresenta edema, o que pode influenciar nos resultados (KALANTAR-ZADEH et al., 2001).

Dentre os marcadores bioquímicos, destaca-se a albumina por ser um potente preditor de morbidade e mortalidade na DRC independentemente das causas que levam à redução da sua concentração sérica. A concentração sérica de albumina deve ser mantida acima de 4,0 g/Dl (CUPPARI; KAMIMURA, 2009).

Recentemente, tem-se dado ênfase à Avaliação Subjetiva Global (ASG), por ser um método simples, de baixo-custo e validado para avaliação do estado nutricional nessa população (VEGINE et al., 2011). Kalantar-Zadeh et al. (2001), criadores da ASG adaptada para pacientes renais, referem que se esse sistema de pontuação abrangente for capaz de estratificar o risco de um paciente com DRC de forma quantitativa, se tornará uma ferramenta prática e fácil sem métodos complicados e cálculos sofisticados. Mais recentemente, Fetter et al. (2014) realizaram a adaptação transcultural da língua 
inglesa para a portuguesa da avaliação global subjetiva de 7 pontos (AGS-7p) e do malnutrition inflammation score (MIS), métodos de avaliação nutricional comumente empregados em pacientes em diálise.

Neste cenário, 0 presente trabalho teve como objetivo correlacionar os métodos de avaliação nutricional em pacientes submetidos à hemodiálise em uma clínica particular do município de São Paulo.

\section{METODOLOGIA}

Trata-se de um estudo de delineamento transversal realizado em uma clínica especializada em doença renal crônica situada no município de São Paulo. A amostra de estudo foi composta por pacientes de ambos os sexos e com idade superior a 18 anos, que realizavam hemodiálise no período da coleta de dados. Foram excluídos pacientes que estiveram internados, que faltaram à sessão de hemodiálise, que iniciaram o tratamento dialítico após o início da coleta de dados ou que não tiveram condições de responder ao questionário.

Para a coleta de dados foi aplicada a Avaliação Subjetiva Global para pacientes com doença renal, adaptada por Kalantar-Zadeh et al. (2001). Tais autores modificaram a AGS original e propuseram uma versão modificada chamada de dialisys malnutrition score, a qual, posteriormente, deu origem ao malnutrition inflammation score (MIS).

O questionário era composto por 10 perguntas, divididas em 4 sessões: Histórico Nutricional, Exame Físico, Índice de Massa Corporal e Exames Laboratoriais e a somatória das perguntas poderia variar de 0 para normal a 30 para desnutrição severa. A primeira sessão continha 5 perguntas adaptadas da Avaliação Subjetiva Global original que contém quatro níveis de severidade: 0 para normal e 3 para severamente anormal.

A mudança de peso foi determinada pela alteração de peso, após a sessão de diálise, nos últimos 6 meses. Score 0 representava perda de até $0,5 \mathrm{~kg}$ ou se houve um aumento de peso; score 1 representava perda de $0,5 \mathrm{~kg}$ a $1,0 \mathrm{~kg}$; score 2 representava perda de pelo menos $1,0 \mathrm{~kg}$, porém menos de $5 \%$ do peso corporal; score 3 representava perda de mais de $5 \%$ do peso corporal.
Para ingestão alimentar foi atribuído o score 0 se a ingestão habitual de alimentos sólidos não sofreu redução na qualidade ou na quantidade; score 1 se a ingestão de alimentos sólidos estava ligeiramente abaixo do ideal; score 2 se a dieta contivesse muito líquido ou houve moderada redução na ingestão de alimentos; score 3 se a ingestão diária de alimentos era incompatível com uma alimentação adequada para a doença.

Nos sintomas gastrointestinais, o score 0 foi atribuído se o paciente encontrava-se com apetite adequado e sem sintomas gastrointestinais; score 1 se houve diminuição do apetite ou leve náuseas; score 2 para vômitos ocasionais ou moderados sintomas gastrointestinais tal como dor abdominal; score 3 para diarreia, vômito frequente ou anorexia severa.

Capacidade funcional apresentava score 0 para capacidade funcional normal ou melhoria considerável do comprometimento funcional anterior; score 1 quando apresentava dificuldade ocasional em deambular ou se sentir cansado com frequência; score 2 quando apresentava dificuldade em desempenhar atividades independentes; score 3 quando apresentava restrição a atividades leves ou acamado e/ou restrito a cadeira de rodas.

A questão sobre comorbidades, que foi acrescentada neste questionário adaptado, é importante, pois o tempo em diálise pode ter uma influência sobre o grau de desnutrição e inflamação. Para a pontuação, utilizou-se score 0 quando o paciente não apresentava outras doenças e foi submetido a hemodiálise há menos de 1 ano; score 1 para comorbidade moderada, excluindo doenças como insuficiência cardíaca congestiva classe III ou IV, doenças coronarianas graves, síndrome da imunodeficiência, doença pulmonar obstrutiva de moderada a grave, e neoplasia metastática (CCMs), ou terapia de diálise de 1 a 4 anos; score 2 também para comorbidade moderada, porém incluindo uma das CCMs ou terapia em diálise há mais de 4 anos; score 3 para duas ou mais CCMs. A existência de diabetes por si só não foi contabilizada se as comorbidades anteriormente listadas não estiveram presentes.

A seção exames físicos foi constituída por dois componentes. Para avaliar a reserva de gordura corporal foram levadas em conta quatro áreas do corpo: abaixo dos 
olhos, tríceps, bíceps e peito. Sinais de perda de massa muscular foram obtidos examinando brevemente sete áreas: têmpora, clavícula, costelas (espaços intercostais), quadríceps, joelho e músculos intraósseos. Para cada um desses dois componentes uma pontuação de 0 a 3 foi atribuída (variando-se de normalidade a mudanças graves), de acordo com as diretrizes da Avaliação Subjetiva Global com base nos critérios já especificados.

Foi adicionada uma função de peso corporal para determinar IMC, utilizando peso pós-diálise e altura ao quadrado e foi classificado de 0 a 3 , representando IMC superior a 20, de 18 a 19,99, de 16 a 17,99 e inferior a $16 \mathrm{~kg} / \mathrm{m}^{2}$ respectivamente.

Na seção de exames laboratoriais incluem-se dois valores, nível de albumina sérica, que é um forte indicador de mortalidade entre os pacientes com insuficiência renal terminal, e transferrina. Esses dois valores compreendem $20 \%$ da pontuação total da avaliação.

Para a determinação dos outros indicadores de desnutrição (Índice de Massa Corporal e aferição de medidas antropométricas), os valores de peso e altura foram coletados nos prontuários do paciente referentes ao mês da coleta de dados. Em seguida, foi calculado o Índice de Massa Corporal através da fórmula IMC $=\mathrm{PESO} /$ ALTURA $^{2}$ e os pacientes com idade entre 18 e 59 anos foram classificados segundo OMS (1995), e segundo OPAS (2002) para pacientes com 60 anos ou mais.

Para aferir a prega cutânea de tríceps foi utilizado um adipômetro da marca Lange e para a circunferência de braço foi utilizada uma fita inextensível de $1,5 \mathrm{~m} \mathrm{e}$ precisão de $0,1 \mathrm{~cm}$ da marca TBW®. Para o cálculo da circunferência muscular de braço foi utilizada a fórmula $\mathrm{CMB}=\mathrm{CB}(\mathrm{cm})-\varpi \times[\mathrm{PCT}(\mathrm{mm}) / 10]$. Estas medidas foram aferidas três vezes e foi considerado o valor médio.

Todas as medidas antropométricas foram aferidas após a sessão de hemodiálise pelas pesquisadoras e os valores dos exames laboratoriais foram coletados no prontuário dos pacientes. Foram considerados exames realizados até 3 meses antes da coleta de dados. Para a classificação das variáveis dos adultos, usou-se a referência de Frisancho (1990), e para classificar os idosos, foram utilizados os padrões de referência de Nhanes III (19881994).

Para a organização dos dados e tabulação das informações coletadas foi utilizado o programa $M S$
Excel e MS Word, versão 2013. A análise da distribuição das variáveis quantitativas demonstrou que algumas não possuíam distribuição normal, segundo teste de Kolmogorov-Smirnov. Por esta razão, foi aplicado o teste de correlação de Spearman, adotando-se o nível de significância de 5\%, utilizando-se o programa estatístico SPSS versão 19 (IBM, 2010). As correlações avaliadas foram IMC vs. Questionário, IMC vs. $\mathrm{CMB}, \mathrm{DCT}$ vs. $\mathrm{CB}$ e CMB vs. Questionário.

Foi garantido aos sujeitos da pesquisa, através do termo de consentimento livre e esclarecido, o direito de se retirar do estudo a qualquer momento ou até se recusar de participar do estudo desde o início, em qualquer uma de suas etapas. Os procedimentos para o desenvolvimento deste estudo respeitaram as diretrizes e normas que regulamentam as pesquisas envolvendo humanos, aprovadas pela Resolução $n^{0}$ 466/2012, do Conselho Nacional de Saúde e o estudo foi aprovado pela Comissão interna de ética em Pesquisa da Universidade Presbiteriana Mackenzie (processo ${ }^{\circ}$ N001/04/14).

\section{RESULTADOS}

A amostra total foi constituída de 87 pacientes, porém foram excluídos 7 pacientes que apresentavam Alzheimer, 15 que se recusaram participar da pesquisa e 5 que passaram mal durante a sessão de hemodiálise, resultando assim em uma amostra final de 60 pacientes.

$\mathrm{Da}$ amostra final 22 indivíduos eram do sexo feminino (36,6\%) e 38 eram do sexo masculino (63,3\%). Da população estudada, 37 eram idosos com idade média de 71,3 anos e 23 eram adultos, com idade média de 46,9 anos.

Diante deste cenário, para a classificação das variáveis Índice de Massa Corporal (IMC), Dobra Cutânea de Tríceps (DCT), Circunferência de Braço (CB) e Circunferência Muscular de Braço (CMB) a amostra foi dividida em dois grupos: Idosos e Adultos (Tabela 1). 
Tabela 1. Média das variáveis antropométricas de pacientes submetidos à hemodiálise em uma clínica particular. São Paulo, 2014

\begin{tabular}{lcccc}
\hline \multirow{2}{*}{ Variável antropométrica } & Feminino & Masculino & & \\
\cline { 2 - 5 } & Adulto & Idoso & Adulto & Idoso \\
\hline $\mathrm{IMC}\left(\mathrm{kg} / \mathrm{m}^{2}\right)$ & 19,92 & 27,39 & 15,2 & 24,1 \\
$\mathrm{CB}(\mathrm{cm})$ & 28,57 & 29,7 & 28,5 & 28,21 \\
$\mathrm{DCT}(\mathrm{mm})$ & 16,85 & 17,66 & 10,11 & 12,9 \\
$\mathrm{CMB}(\mathrm{cm})$ & 23,27 & 24,15 & 25,32 & 24,16 \\
\hline
\end{tabular}

A Tabela 2 apresenta a distribuição percentual de pacientes submetidos à hemodiálise em uma clínica particular segundo estado nutricional.

Tabela 2. Distribuição percentual de pacientes submetidos à hemodiálise em uma clínica particular segundo estado nutricional. São Paulo, 2014

\begin{tabular}{lccccc}
\hline Variável antropométrica & Desnutrição (\%) & Risco nutricional (\%) & Eutrofia (\%) & Sobrepeso (\%) & Obesidade (\%) \\
\hline IMC $\left(\mathrm{kg} / \mathrm{m}^{2}\right)$ & 36,6 & - & 45,0 & 11,7 & 6,7 \\
CB $(\mathrm{cm})$ & 43,3 & 8,3 & 43,3 & 3,4 & 1,7 \\
DCT $(\mathrm{mm})$ & 6,6 & 30,0 & 50,0 & 11,7 & 1,7 \\
CMB $(\mathrm{cm})$ & 25,0 & 25,0 & 31,6 & 11,7 & 6,7 \\
\hline
\end{tabular}

Outra variável do estudo foi a pontuação final do questionário, aplicado para todos os indivíduos participantes da pesquisa. A média de pontos para o grupo de idosos foi 9,7 e para o grupo dos adultos foi de 6,6 .

No momento da coleta de dados não foi localizado no prontuário dos pacientes o valor de transferrina, um dos exames laboratoriais utilizados no questionário, porém o estudo teve continuidade excluindo-se apenas a questão 10.
Uma das categorias do questionário tratava de comorbidades, sendo que as mais frequentemente relatadas foram: hipertensão $(76,6 \%)$, diabetes $(45 \%)$, dislipidemia (21\%), insuficiência cardíaca congestiva $(13,3 \%)$ e outras comorbidades $(18,3 \%)$.

Os coeficientes de correlação de Spearman e valor p entre variáveis antropométricas dos pacientes estão expressos na Tabela 3.

Tabela 3. Coeficientes de correlação de Spearman e valor p entre variáveis antropométricas de pacientes submetidos à hemodiálise em uma clínica particular. São Paulo, 2014

\begin{tabular}{lccr}
\multicolumn{1}{c}{ Correlação } & Coeficiente de correlação de Spearman & Valor $\boldsymbol{p}$ & Intensidade da correlação \\
\hline IMC vs. ASG adaptada & 0,089 & 0,501 & Muito fraca \\
IMC vs. CMB & 0,548 & 0,000 & Fraca \\
DCT vs. CB & 0,416 & 0,001 & Fraca \\
CMB vs. ASG adaptada & 0,023 & 0,862 & Muito fraca \\
\hline
\end{tabular}


As correlações entre IMC vs. questionário e CMB vs. questionário não apresentaram significância estatística, sendo que este fato pode ter ocorrido por ter sido desconsiderada a questão 10 do questionário. Para IMC e CMB a intensidade de correlação foi fraca, porém com significância estatística, o que ocorreu igualmente com as variáveis DCT vs. $\mathrm{CB}$.

\section{DISCUSSÃO}

Este trabalho avaliou a correlação dos métodos de avaliação nutricional em pacientes com doença renal crônica em tratamento de hemodiálise. Os resultados obtidos mostraram correlações fracas ou muito fracas de acordo com as variáveis, o que parece demonstrar que, para o diagnóstico de risco nutricional neste grupo, o uso de apenas um método pode não detectar a presença de depleção energético-proteica, podendo levar a erro no diagnóstico nutricional.

Com relação aos dados de idade e sexo, o presente trabalho diferencia-se do estudo de Oliveira et al. (2010), sendo que a média de idade foi de 49,22 anos, $51,7 \%$ eram do sexo feminino e $48,3 \%$ masculino. Também difere-se do estudo de Koehnlein, Yamada e Giannasi (2008), em que 61\% eram adultos e 38\% eram idosos. Estes dados podem ter se diferenciado pelo bairro em que se encontra o local da pesquisa, reconhecido por sua alta prevalência de idosos.

Em um estudo realizado no Estado de Santa Catarina (CARDOZO; VIEIRA; CAMPANELLA, 2006), verificou-se no total de avaliados um pequeno percentual de desnutridos e um elevado índice de sobrepeso, com mais de $50 \%$ dos indivíduos em eutrofia. No presente estudo, a maior parte da amostra encontrava-se eutrófica, porém foram observados $36 \%$ de desnutridos. Vegine et al. (2011), em trabalho realizado com pacientes em tratamento crônico de hemodiálise de um hospital universitário, encontraram eutrofia segundo $\mathrm{CB}$ no grupo feminino e um grau indicativo de desnutrição leve no grupo masculino.

Em relação ao IMC, $45 \%$ da amostra estudada se mostrou eutrófica, o mesmo encontrado no estudo proposto por Koehnlein, Yamada e Giannasi (2008), que apresentou 57,8\% e 50\% de eutrofia na população adulta e idosa respectivamente. Já para a variável CMB, o estudo proposto por Koehnlein, Yamada e Giannasi (2008) demonstrou que $28,57 \%$ das mulheres e $42,83 \%$ dos homens apresentaram desnutrição leve ou algum grau de desnutrição. No presente estudo, em relação à mesma variável, $25 \%$ dos indivíduos se mostraram desnutridos ou em risco nutricional.

No presente estudo, os pacientes foram classificados, segundo a pontuação do questionário, em 3 grupos: de 0 a 9 pontos como eutróficos, de 9,1 a 18 pontos sob risco nutricional e de 18,1 a 27 pontos como desnutridos. Sob este critério, observou-se apenas que os pacientes idosos apresentaram risco nutricional, o que difere do estudo proposto por Oliveira et al. (2010), em que $94,8 \%$ do total de pacientes estavam sob risco nutricional/desnutrição leve.

Outros estudos também avaliaram as comorbidades mais presentes, apresentando como principais: hipertensão, diabetes e glomerulonefrite. No estudo proposto por Santos et al. (2006) relatou-se que a comorbidade mais frequente que desencadeia a doença renal é a glomerulonefrite (42,7\%) e a hipertensão como subsequente (34\%). Oliveira et al. (2012) relataram que $89,4 \%$ apresentaram hipertensão e 35,1\% apresentaram diabetes. Um fato relevante desse estudo constatou que os pacientes hipertensos apresentaram de 0,2 a 0,8 vezes menos probabilidade de ter desnutrição.

Diversos autores como Sant'Anna, Priore e Franceschini (2009) e Oliveira et al. (2010) utilizaram correlação como instrumento de seus estudos. Estes relatam que há uma dificuldade na correlação entre métodos qualitativos e quantitativos e que indicadores isolados não apresentam boa correlação. O mesmo foi notado no presente estudo, em que se avaliou os pacientes quantitativamente e qualitativamente, resultando em uma baixa intensidade de correlação.

Sendo assim, apesar da existência de diversos métodos para avaliação nutricional de pacientes submetidos à hemodiálise, cada um apresenta vantagens e desvantagens. Desta forma, ao avaliar um paciente nestas condições deve-se atentar ao método que melhor se encaixa às necessidades individuais. 


\section{CONCLUSÃO}

A intensidade das correlações entre as variáveis IMC vs. ASG adaptada, IMC vs. CMB, DCT vs. CB e CMB vs. ASG adaptada foi muito fraca, fraca, fraca e muito fraca respectivamente. Destaca-se que as correlações IMC vs. $\mathrm{CMB}$ e DCT vs. $\mathrm{CB}$, apesar de fracas, se mostraram significativas.

Pode-se concluir que o uso de diferentes métodos de avaliação nutricional, com variáveis subjetivas e objetivas, representa significativa melhoria do padrão diagnóstico de pacientes com DRC. Deve-se sempre levar em consideração custos, nível de treinamento dos avaliadores, tempo de execução, receptividade da população e possíveis riscos à saúde para definir o melhor método. É imprescindível, ainda, que o método escolhido seja validado para a população a ser estudada.

\section{REFERÊNCIAS}

BIAVO, B. M. M.; TZANNO-MARTINS, C.; CUNHA, L. M.; ARAÚJO, M. L.; RIBEIRO, M. M. C.; SACHS, A.; UEZIMA, C. B. B.; DRAIBE, S. A.; RODRIGUES, C. I. S.; BARROS, E. J. G. Aspectos nutricionais e epidemiológicos de pacientes com doença renal crônica submetidos a tratamento hemodialítico no Brasil. J Bras Nefrol, n. 34, v. 3, p. 206215, 2012.

BIGOGNO, F. G.; FETTER, R. L.; AVESANI, C. M. Aplicabilidade da avaliação global subjetiva e malnutrition inflammation score na avaliação do estado nutricional na doença renal crônica. J Bras Nefrol, v. 36, n. 2, p. 236240, 2014.

CARDOZO, M. T.; VIEIRA, I. O.; CAMPANELLA, L. C. A. Alterações nutricionais em pacientes renais crônicos em programa de hemodiálise. Rev Bras Nutr Clin, v. 21, n. 4, p. 284-289, 2006.

CUPPARI, L.; KAMIMURA, M. A. Avaliação nutricional na doença renal crônica: desafios na prática clínica. J Bras Nefrol, v. 31, n. 1, p. 28-35, 2009.

FAVA, S. M. C. L.; OLIVEIRA, A. A.; VITOR, E. M.; DAMASCENO, D. D.; LIBÂNIO, S. I. C. Complicações mais frequentes relacionadas aos pacientes em tratamento dialítico. Rev. Min. Enf., v. 10, n. 2, p. 145-150, 2006.
FETTER, R. L.; BIGOGNO, F. G.; OLIVEIRA, F. G. P.; AVESANI, C. M. Adaptação transcultural para o português de instrumentos de avaliação do estado nutricional de pacientes em diálise. J Bras Nefrol. v. 36, n. 2, p. 176$185,2014$.

FRISANCHO, A.R. Anthropometric Standards for the Assessment of Growth and Nutritional Status. Michigan: University of Michigan Press, 1990.

IBM Corp. Released 2010. IBM SPSS Statistics for Windows, Version 19.0. Armonk, New York: IBM Corp.

KALANTAR-ZADEH, K.; KOPPLE, J. D.; BLOCK, G.; HUMPHREYS, M. H. A malnutrition-inflammation score is correlated with morbidity and mortality in maintenance hemodialysis patients. American Journal of Kidney Diseases, v. 38, n. 6, p. 1251-1263, 2001.

KAMIMURA, M. A.; BAZANELLI, A. P.; CUPPARI, L. Nutrição em Diálise Peritoneal. In: VIEIRA NETO, O. M.; ABENSUR, H. (Org.). Diálise Peritoneal: Manual Prático. São Paulo: Livraria Balieiro, 2013, v. 1, p. 75-103.

KAMIMURA, M. A.; BAZANELLI, A. P.; CUPPARI, L. Nutrição em diálise peritoneal. In: VIEIRA NETO, O. M.; ABENSUR, H. (Org.). Diálise peritoneal - Manual prático. São Paulo: Livraria Balieiro, 2012. p. 75-103.

KAMIMURA, M. A.; DRAIBE, S. A.; SIGULEM, D. M.; CUPPARI, L. Métodos de avaliação da composição corporal em pacientes submetidos à hemodiálise. Rev Nutr, Campinas, v. 17, n. 1, p. 97-105, 2004.

KIRSZTAJN, G. M.; BASTOS, M. G.; ANDRIOLO, A. Dia Mundial do Rim 2011 - Proteinúria e creatinina sérica: testes essenciais para diagnóstico de doença renal crônica. J Bras Patol Med Lab, n. 47, v. 2, p. 100-3, 2011.

KOEHNLEIN, E. A.; YAMADA, A. N.; GIANNASI, A. C. B. Avaliação do estado nutricional de pacientes em hemodiálise. Acta Sci. Health Sci., v. 30, n. 1, p. 65-71, 2008.

NATIONAL KIDNEY FOUNDATION. Kidney Disease Outcomes Quality Initiative. Clinical practice guidelines for chronic kidney disease: evaluation, classification and stratification. Am J Kidney Dis, n. 39, v. 1, p. S1-266, 2002. 
NERBASS, F. B.; CUPPARI, L. Hemodiálise. In: CUPPARI, L.; AVESANI, C. M.; KAMIMURA, M. A. Nutrição na doença renal crônica. Barueri: Manole, 2013. p. 247-69.

OLIVEIRA, C. M. C.; KUBRUSLY, M.; MOTA, R. S.; SILVA, C. A. B; OLIVEIRA, V. N. Desnutrição na insuficiência renal crônica: qual o melhor método diagnóstico na prática clínica? J Bras Nefrol, n. 32, v. 1, p. 57-70, 2010.

OLIVEIRA, G. T. C.; ANDRADE, E. I. G.; ACURCIO, F. A.; CHERCHIGLIA, M. L.; CORREIA, M. I. T. D. Avaliação nutricional de pacientes submetidos à hemodiálise em centros de Belo Horizonte. Rev Assoc Med Bras, v. 58, n. 2, p. 240-247, 2012.

ORGANIZACIÓN PANAMERICANA DE LA SALUD - OPAS. SABE: Survey on Health and Well-Being of Elders: preliminary report. Washington D.C.: Organización Panamericana de la Salud, 2002.

SANT'ANNA, M. S.; PRIORE, S. E.; FRANCESCHINI, S. D. C. Métodos de avaliação da composição corporal em crianças. Ver Paul Pediatr, v. 3, n. 27, p. 315-321, 2009.

SESSO, R. C.; LOPES, A. A.; THOMÉ, F. S.; LUGON, J. R.; WATANABE, Y.; SANTOS, D. R. Relatório do Censo Brasileiro de Diálise Crônica 2012. J Bras Nefrol, n. 36, v. 1, p. 48-53, 2014.

SANTOS, P.R.; COELHO, M.R.; GOMES, N.P.; JOSUÉ, C. E. $P$. Associação de indicadores nutricionais com qualidade de vida em pacientes portadores de doença renal crônica em hemodiálise. J Bras Nefrol, v.28, n. 2, p. 57 - 64, 2006.

VEGINE, P. M.; FERNANDES, A. C. P.; TORRES, M. R. S. G.; SILVA, M. I. B.; AVESANI, C. M. Avaliação de métodos para identificar desnutrição energético-proteica de pacientes em hemodiálise. J Bras Nefrol, v. 33, n. 1, p. 55-61, 2011.

WORLD HEALTH ORGANIZATION (WHO). Physical status: the use and interpretation of anthropometry. Report of a WHO Expert Committee. WHO Technical Report Series 854. Geneva, World Health Organization, 1995.
Recebido em: 16 de junbo de 2015 Aceito em: 24 de agosto de 2015 